\title{
Complications of Drug Treatment and Measures to Eliminate Them
}

\author{
Salimova NoilaY unusovna* \\ Student at the Samarkand institute of veterinary medicine, Russia \\ *Corresponding author: SalimovaNoilaYunusovna, Student at the Samarkand institute of veterinary medicine, Russia
}

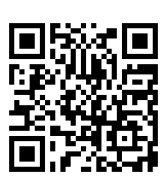

\section{ARTICLE INFO}

Received: 絊 July 27, 2020

Published: August 07, 2020

Citation: Salimova Noila Yunusovna. Complications of Drug Treatment and Measures to Eliminate Them. Biomed J Sci \& Tech Res 29(3)-2020. BJSTR. MS.ID.004792.

\section{ABSTRACT}

The article contains information from the literature about possible side effects of various medicines used in medical and veterinary practice, as well as measures to eliminate them.

Keywords:Drug; Local; Reflex; Toxic; Antibiotic; Teratogen; Embryotoxic; The Mutagen; Cancerogen; Allergen

\section{Introduction}

The harmful effect of drugs by the transition period is short, medium and long-lasting. These effects occur in the form of shortterm pain, dermatitis, inflammation of the mucous membrane or severe numbness (shock), which sometimes causes deep changes in organs and tissues, and even a tragedy. Most often, these manifestations occur as a result of repeated use of medications. The gradual increase in sensitivity by the body and the occurrence of unforeseen circumstances lead to this. There are many similar terms of the response reaction, which are observed as a result of the action of drugs, and they are all considered poisonous, secondary, negative and side effects, complications of treatment with drugs.In laboratory conditions, the side effects of drugs obtained by chemical synthesis are more likely to be compared to the harmful effects of phytopreparations obtained from medicinal plants.The side effects of many medicinal plants have not been identified. Currently, more than 500 drugs have been identified to reduce the activity of the liver, kidneys, blood-forming organs. These are examples of sulfonamides and some antibiotics (tetracycline, strychomycin) [1].

\section{Types of Adverse Effects of Drugs are As Follows}

Local adverse effects-occur due to the connection of the drug with the tissue (especially the oshozon-intestinal mucosa), as a result of which tissue damage is observed, wounds, erosions, inflammation. Basically, such an effect is characteristic of drugs that irritate the mucous membranes of the gastrointestinal tract (aspirin, indocin, butadione, reserpine, etc.).).This effect is compensated by the fact that the drugs are enveloped in a liquid or shell (capsule), sometimes added With Protein, Milk, starch slime or consumed after a meal (chloralgidrate, butadione, reserpine, acetylsalicylic acid, etc.).The reflex is a negative effect-due to this effect, the activity of the members can be slowed down or vice versa. This effect is sometimes expressed in a positive way. For example, when smelling of novshadil alcohol, there is a weakening of breathing (positive), when narcotic substances smell, shortness of breath (negative), narrowing of bronchi.Toxic effects can be common. This effect occurs due to the intake of large doses of drugs into the body. Quinine has a large dose of hearing loss, irreversible decreasetiradi; monomycin and strychomycin from antibiotics accumulate in high amounts in the inner ear, irreversibly decrease hearingtiradi; sulfanilamides form stones in the kidneys, demonstrating the property of digitoxin cumulation from cardiac glycosides, disrupting cardiac activity[2].

The course of poisoning can be acute and chronic. For example, such cases can be observed in poisoning with morphine, ethyl alcohol, heavy metals (mercury). [3]Dysbacteriosis is a violation of the natural composition of intestinal microorganisms. Useful 
(saprophyte) is the destruction of flora and the outbreaks of the development of harmful (patogen) bacteria. This can also occur in patients who are exhausted and exhausted, after chronic diseases, sometimes as a result of taking corticosteroids, drugs that have a wide spectrum of action against a wide range of bacteria. Candidomycosis-is the basis for the development of a gang of fungi that are characteristic of the type of Candida, on the gastrointestinal mucosa, caused by the use of extensive antibiotics (tetracycline, levomycetin) during treatment. In such cases, it is recommended to use special antibiotics (Nystatin, levorin).Teratogen effect (teratos) - is caused by the intake of certain medications during pregnancy, causing changes in the fetus [1,2].

In 1961 year, as a result of the use of the drug Talidomideas a calming drug by pregnant women, it was found that the fetus is born with various physical defects (the hipness of the limbs, defects in the digestive tract, etc.).the G.). For this reason, from the period of the onset of pregnancy, special attention is paid to the teratogen effect of the drug, special examinations are performed.Especially the central nervous system is extremely sensitive to the teratogen effects of drugs. Mental retardation occurs due to low memory, impaired DNA and RNA activity, sluggishness of neuronlararo relationships, the same effect manifests itself in nature.

To prevent this effect, pregnant women, during the first three months of pregnancy, such drugs are not recommended. The use of drugs in the second half of pregnancy can disrupt the activity of some organs, even if the negative states do not come. Therefore, pregnant women are advised to take the drug in small doses and for a short time, if necessary. Teratogen effect of drugs does not pass from generation to Generation [4].

Embryotoxic effect-occurs under the influence of the drug, which was taken in the initial period of fertilization of the mother egg cell. In this case, the process of adhesion (implantation) of the fertilized cell to the mucous membrane of the uterus (1-2 weeks) and the formation of a placenta (3-6 weeks) is disrupted, spontaneous miscarriage (abortion) of the embryo is observed. This condition can occur when a large dose of the drug is consumed. Basically, the drug occurs when it is absorbed into the embryo, passing through the mucous membrane of the uterus. If the miscarriage (abortion) of the fetus is not observed, the embryotoxic effect, teratogen can cause the effect to occur.Mutagaenetic effect-hereditary (genetic) effect (mutatio-change, genos-generation).it can occur in the period of gonadogenesis or embryogenesis by men or women, due to the reception of medicines. The consequences of this effect can go from generation to generation. Under the influence of drugs that are used against malignant tumors, a change in the structure of DNA leads to the fact that mutagenic changes occur[3-5].

Blastomogen (konserogen) effect (blastoma-dangerous usma)the occurrence of such an effect can be observed as a result of the long-term use of certain chemicals. For example, nicotine in tobacco, benzopyrine, in addition nitrosamines, drugs that suppress nitrobirics, under certain conditions can cause dangerous tumors or accelerate their development.Idiosyncrasy-after the first reception of certain drugs, various strong response reactions are manifested, which are preserved for a lifetime. This condition is explained by enzymopathies that pass from generation to generation (the absence of certain enzymes in the body).An allergy to drugs is a response - unpleasant reaction that occurs when a medicinal product is introduced into the body. The substance of the drug is an immature antigen, which, when combined with proteins in the body, turns into a complete antigen. After 7-12 days against it, lymphocytes produce antibodies, after which a strong allergic reaction may occur. During this reaction, a large number of biologically active substances (histamine, serotonin, bradykinin, prostaglandins) are produced from the cells.

These agents manifest allergic changes that are observed in the body. Such a reaction occurs at a speed, in the case where this drug is sent to the slurry. In some cases, allergic reactions can occur at the same speed when taking the drug for the first time to lose weight. This occurs due to an increase in the body's predisposition. An allergic reaction to fast dressing is regarded as the result of sensibilization. In particular, the reactions that occur against the venom of animals are in the sentence shular. It is regarded as changes that occur due to late-formed reactions, conjugation (the transfer of organs from one person to another).In some cases, the allergy caused by medications begins slowly and lasts for a long time. This condition can persist for a certain period of time even after the introduction of a medicinal product into the body is stopped. For example: means that are used to treat tuberculosis and eczema can cause chronic dermatitis (rash of the ass, rashes).

An allergic reaction to medications can develop in some cases in the form of anaphylactic hangover (shock), which quickly rushes to sleep. As a result, there is a lack of air, a cardiac colic, a decrease in arterial pressure, general malaise and other changes occur. The use of anti-histamine drugs (dimedrol, suprastin), normalizing blood pressure, sending blood tomirga noradrenaline is one of the necessary measures. Such a state of hangover often occurs when sending preparations of the penicillin group from antibiotics. Therefore, in order to prevent such negative phenomena, an allergic test is performed on the Max, that is, a small amount of the antibiotic $(0,1-0,2 \mathrm{ml})$ is injected under the skin to clarify the sensitivity of the organism. Dressing blisters with redness and swelling in the place of shipment, do not send this medication [5;74-76 p.].In order to prevent these side effects, individual (individual) sensitivity to the drug is checked: tabl 1:10 or 1:5 part of the test is poured under the tongue, 15 minutes are observed. If an adverse response occurs, it is not recommended to use the drug. To check the compatibility of the body with drugs introduced through blood vessels, first 0,1-0,2 $\mathrm{ml}$ of solution is introduced, the general condition of the patient is observed, then the rest of the solution is injected. 
Complications, side effects of antibiotics are diverse Complications, which occur as a result of their direct toxic effect, are manifested mainly in the nervous system (visual nerve, polyneuritis, ataxia and others), excretory organs (nephrosis), liver (parenchymatous jaundice), gastrointestinal tract (ogric, diarrhea and others). Often such cases are caused by preparations of strychomycin, neomycin.One of the complications that occur due to tetracycline is teratogen effect. In the period of Xomiladorlik, the development of tumors in the course of its antibiotic effect is disrupted, and the child is born with signs of disability. Another of the complications that are observed due to antibiotics is a case of allergies. Allergy can manifest differently (on the skin, mucous cavities and khakozo). The most dangerous of these types of complications is anaphylactic shock, it is caused mainly by penicillin gel (falling of mining pressure, coronging of es-Hus and others). Therefore, in the first administration of penicillin, a condition is established, which determines the sensitivity of the patient's body to antibiotics.Oxytetracycline-harmful to liver activity and taking into account the effects of teratogen in the first half of pregnancy and when prescribing to children, it is necessary to be very extiotic.

As mentioned above, tetracyclines accumulate in the bone tissue, which can adversely affect the incorrect formation of them, the correct growth of teeth.Tetracycline group of drugs have a pronounced effect on the metabolism processes of the body: the synthesis of oxyl decreases, the excretion of water, sodium, amino acids and some vitamins from the body accelerates. In the long-term stagnation okibati, useful saprophytes in the intestinal microflora are riddled, which can cause the appearance of a family of bacteria that are stagnant in relation to exactly the same group of drugs. As a result, candidomycosis is observed when the disease progresses, sometimes symptoms such as lack of vitamins of Group V are manifested. During the treatment, it is much more effective to dilute the preparations with Nystatin and vitamins of Group P.We will need to focus on the fact that levomycetin has specific side effects. Allergic reactions during treatment, a violation of the activity of the excretory organs that generate mining, can sometimes lead to a tragic ending anemia condition. At the same time, candidomycosis disease is also observed. The short duration of treatment with the drug maxad is suitable.

Antibiotics against fungi. As a result of prolonged use of antibiotics with a wide spectrum of action (Oxicon, strychomycin, levomycetin and others), there is a cavity in the mouth, mucous membranes of the gastrointestinal tract, gangs of fungi belonging to the Candida family; that is, candidomycosis disease occurs. Sometimes these fungi migrate to the internal organs of the blood vessel, which can also cause severe diseases. For the treatment of such diseases, antibiotics such as Nystatin and levorin are recommended. Nystatin-is acidic and rapidly decomposes in the alkaline medium, has insoluble properties in water and alcohol. Therefore, not absorbed into the cone, the load in the intestinal cavity accumulates in microns. It is recommended to apply an hour before meals. To gum from the action of hydrochloric acid is crushed in special cubes (capsules). It can be applied for two weeks, less often and does not cause side effects. In fungal diseases of the skin, $4 \%$ of the drug is recommended ointment li.

Levorin-sodium tuzi of the drug is water-soluble, and at 500000 TBETETS are recommended 2-3 times a day for 10-12 days, during the night. In the case of candidomycosis of the oral cavity, there are special napkins that are designed to hold in the mouth. As a result of this, the accumulation of the drug in the oral cavity of the pharynx is observed. For puddling on the surface, ointments were also prepared.Pesticides used in veterinary practice are also manifested. They have properties of concomitantogen (the occurrence of cancer cells), teratogen (negative influence on heredity), mutagen (manifestation of mutilation, disorder), embryotoxic (toxic effect on the fetus), allergic effect. These cases are manifested when poisoning from the action of phosphoric and chlorogenic compounds, carbs, artificial pyrethroids, which belong to the group of more pesticides, occurs. Similar properties are observed in them, which are hemotoxic, hepatoxic, nephrotoxic, allergic, traumatizing membranes, as well as inhibiting the activity of cholinesterase, which manifests its harmful effect on the body.Strict compliance with the rules of storage, transportation and application of these tools for the purpose of prevention and treatment of such adverse effects is one of the main requirements. When poisoning occurs, primary antidote therapy, that is, it is advisable to apply anti-poison agents ( atropine sulfate,cocorbaxylase, tropacin, fagangan coal, vikasol, unitiol ,siysiy chloride, thiosulfate sodium,methylamine, potassium permanganate, tannin, glucose) on time, and then conduct symptomatic, etiotropic and pathogenetic treatment procedures.

\section{References}

1. Baltkais YY, Fateev VA (1991) Interaction of medicinal substances. Moscow, Meditsina.

2. Zhulenko VN (2004) Veterinary toxicology. Moscow, Koloss.

3. Salimov Y (2019) Veterinary pharmacology. Tashkent, Noshir.

4. Sokolova VD (2010) Pharmacology. Saint Petersburg. Moscow Krasnodar.

5. Subbotnik VM (2000) Modern medicines in veterinary medicine. RostovOn-Don, Phoenix 
ISSN: 2574-1241

DOI: $10.26717 /$ BJSTR.2020.29.004792

Salimova Noila Yunusovna. Biomed J Sci \& Tech Res

(cC) This work is licensed under Creative

Submission Link: https://biomedres.us/submit-manuscript.php

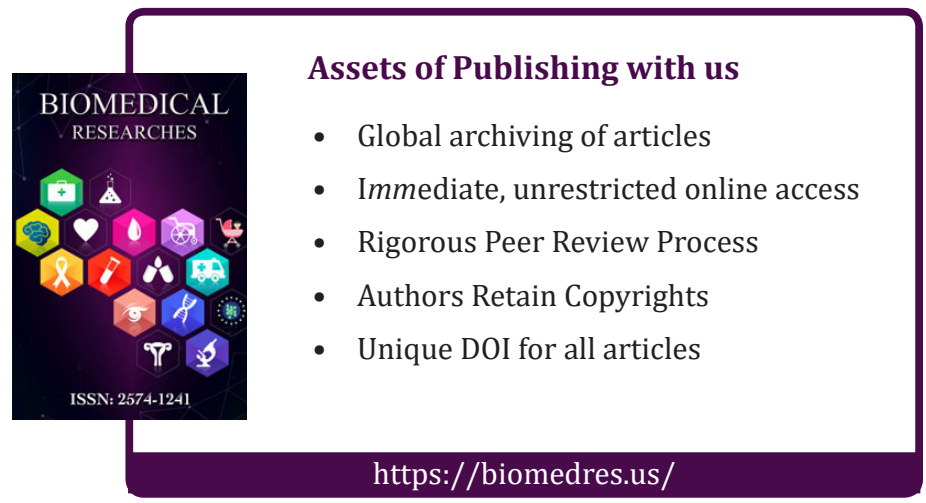

